\title{
Hyaluronate - Black Phosphorus Conjugates as a Copper Chelating Agent for Wilson Disease Treatment
}

\section{Seong-Jong Kim}

Pohang University of Science and Technology

Hye Hyeon Han

Pohang University of Science and Technology

Sei Kwang Hahn ( $\nabla$ skhanb@postech.ac.kr)

Pohang University of Science and Technology https://orcid.org/0000-0002-7718-6259

\section{Research Article}

Keywords: Black phosphorus nanosheets, Hyaluronate, Chelating agent, Wilson disease

Posted Date: May 26th, 2021

DOl: https://doi.org/10.21203/rs.3.rs-524403/v1

License: (c) (i) This work is licensed under a Creative Commons Attribution 4.0 International License.

Read Full License 


\section{Hyaluronate - Black Phosphorus Conjugates as a Copper Chelating Agent for Wilson Disease Treatment}

Seong-Jong Kim†, Hye Hyeon Han†, and Sei Kwang Hahn*

\section{*Correspondence:}

skhanb@postech.ac.kr

Department of Materials Science

and Engineering, Pohang

University of Science and

Technology (POSTECH), 77

Cheongam-ro, Nam-gu, Pohang,

Gyeongbuk 37673, Korea

† These authors contributed

equally to this work and should

be considered as co-first authors

\begin{abstract}
Background: Wilson disease (WD) is a genetic disorder of copper storage, resulting in pathological accumulation of copper in the body. Because symptoms are generally related to the liver, chelating agents capable of capturing excess copper ions after targeted delivery to the liver are highly required for the treatment of WD.

Methods: We developed hyaluronate - black phosphorus (HA-BP) conjugates for capturing copper ions accumulated in the liver for the treatment of WD.

Results: HA-BP conjugates showed high hepatocyte-specific targeting efficiency, selective copper capturing capacity, excellent biocompatibility, and biodegradability. HA enhanced the stability of BP nanosheets and increased copper binding capacity. In vitro cellular uptake and competitive binding tests verified targeted delivery of HA-BP conjugates to liver cells via HA receptor mediated endocytosis. The cell viability test confirmed the high biocompatibility of HA-BP conjugates.
\end{abstract}

Conclusion: HA-BP conjugates would be an efficient copper chelating agent to remove accumulated copper in the liver for the WD treatment.

Keywords: Black phosphorus nanosheets, Hyaluronate, Chelating agent, Wilson disease 


\section{Background}

Wilson disease (WD) is an inborn disorder of copper metabolism and characterized by copper overload in the organs, especially liver and brain [1]. In patients with WD, copper cannot be eliminated properly, causing liver cirrhosis and liver transplantation in severe cases [2]. Accordingly, WD is one of the most challenging diseases in medicine. There are two available treatments to increase copper excretion and reduce copper absorption by using (1) chelators and (2) zinc salts [3]. Combination therapy using zinc salts and chelators leads to clearing copper overloaded tissues and blocking the pathological accumulation of copper in the organs $[4,5]$. However, the long-term use of the medication is limited because of the safety issues and severe adverse effects [6]. Chelators, such as D-penicillamine and trientine, have been reported to cause adverse events like marrow toxicity, lupus-like syndrome, and anemia [7, 8]. As a suitable chelator, the agent should be able to efficiently capture excess transition-metal ions with excellent biocompatibility and biodegradability. Recently, various nanomaterials have been widely investigated as novel chelators to form nontoxic metal complexes [9]. In addition, nanoparticlemediated drug delivery efficiently reduced several toxic metals in the body [10]. However, there are few in-depth studies focusing on targeted delivery of chelating agents to the liver for the treatment of WD.

Here, we developed a hyaluronate - black phosphorus (HA-BP) conjugate to remove accumulated copper, especially in the liver. Black phosphorus (BP) nanosheets are well known as a biodegradable 2D material composed of phosphorus atoms $[11,12]$. Due to its great biocompatibility, BP nanosheets have been used for biomedical applications including phototherapy, drug delivery, and biocatalysis [13]. Furthermore, phosphorus binds strongly with metal ions, especially $\mathrm{Cu}^{2+}$, making BP nanosheets a robust nanocaptor for copper ions [9]. To prevent the rapid degradation of BP nanosheets from oxidation, BP nanosheets were coated with HA [14]. HA is a natural biodegradable polymer with a high binding affinity toward liver cells $[15,16$, $17,18]$. After the physicochemical characterization of HA-BP conjugates, we investigated the copper capturing capability, biocompatibility and biodegradability via in vitro liver-specific targeted delivery of HA-BP conjugates for WD treatment.

\section{Methods}

Materials

Bulk crystals of black phosphorus (BP) were obtained from Smart-elements (Vienna, Austria). Sodium hyaluronate (HA, MW = $10 \mathrm{kDa}$ ) was acquired from Lifecore Biomedical (Chaska, MN, USA). 1-Methyl-2-pyrrolidinone (NMP), 1,6diaminohexane (DAH), dimethyl sulfoxide (DMSO), and $\mathrm{N}$ hydroxysulfosuccinimide (NHS) sodium salt were purchased from Sigma Aldrich (St. Louis, MO). 5-Aminofluorescein (FITC) and 1-ethyl-3-(3- 
(dimethylamino)propyl)carbodiimide (EDC) hydrochloride were purchased from Tokyo Chemical Industry (Tokyo, Japan). Cell counting kit-8 (CCK-8) was acquired from Dojindo Molecular Technologies (Kumamoto, Japan). Dulbecco' s modified Eagle' s medium (DMEM), fetal bovine serum (FBS), and antibiotics were purchased from Gibco (Grand Island, NY). HepG2 cells were purchased from Korean Cell Line Bank (Seoul, Korea).

Preparation of BP nanosheets

BP nanosheets were prepared by liquid exfoliation technique. In brief, $30 \mathrm{mg}$ of $\mathrm{BP}$ was dispersed in $30 \mathrm{~mL}$ NMP solution with sodium hydroxide. Then, the solution was sonicated for $10 \mathrm{~h}$ (amplifier: $20 \%$, on/off: $6 \mathrm{~s} / 4 \mathrm{~s}$ ). The nonexfoliated BP nanosheets were removed using centrifugation for $10 \mathrm{~min}$ at $5,000 \mathrm{rpm}$ and the supernatant was centrifuged again for $15 \mathrm{~min}$ at $12,500 \mathrm{rpm}$ to re-disperse BP nanosheets in distilled (DI) water.

Preparation of HA-BP conjugates

HA-DAH was synthesized by the EDC chemistry between HA (10 kDa, $100 \mathrm{mg}$ ) and DAH (578 mg, 10 molar ratios to $\mathrm{HA}$ ) in DI water at $\mathrm{pH} 4.8$ for $24 \mathrm{~h}$. Then, HA-DAH was dialyzed against $15 \%$ ethanol and DI water, respectively (MWCO $=3,500 \mathrm{Da}$ ). HA-DAH was freeze-dried for 3 days. After that, BP nanosheets were mixed with HA-DAH at an equal content and stirred for $1 \mathrm{~h}$ to form HAmodified BP (HA-BP). The solution was centrifuged for $15 \mathrm{~min}$ at 12,500 rpm and supernatant containing $\mathrm{HA}-\mathrm{BP}$ conjugates was collected to remove excess HA-DAH.

Characterization of BP nanosheets and HA-BP conjugates

The prepared BP nanosheets and HA-BP conjugates were analysed by dynamic light scattering (DLS, Zetasizer Nano ZS90, Malvern Instruments Co., Malvern, UK), UV/vis spectrophotometry (S-3100, Scinco Co., Seoul, Korea), Fourier transform - infrared spectroscopy (FT-IR, Cary 600, Agilent Technologies), and transmission electron microscopy (TEM, JEM-1011, JEOL Co., Akishima, Japan). The physical structure of BP nanosheets and HA-BP conjugates was analysed by TEM, and the surface modification of BP nanosheets with HA was assessed by DLS and FT-IR.

Stability and biodegradation tests

BP nanosheets and HA-BP conjugates were suspended in DI water at an equal concentration and then the absorbance spectrum of each solution was measured for 7 days by UV/vis spectrophotometry.

In vitro release test of FITC

First, HA-FITC conjugates were synthesized by the EDC chemistry between FITC and HA-DAH with a DAH content of $50 \mathrm{~mol} \%$. BP-FITC conjugates were prepared by simple mixing of BP nanosheets and FITC, and HA-BP-FITC conjugates were synthesized by the EDC chemistry between BP nanosheets and 
HA-FITC. After 0, 48, 96, and $144 \mathrm{~h}$, the solutions were centrifuged for $15 \mathrm{~min}$ at $12,500 \mathrm{rpm}$ and the supernatants were collected to remove excess FITC or HAFITC. Fluorescent intensity of BP-FITC and HA-BP-FITC conjugates was analyzed for 7 days using a microplate fluorometer (Fluoroskan ascent FL, Thermo Scientific). The released amount of FITC was calculated from the decrease of the fluorescent intensity.

Determination of $\mathrm{Cu}$ content $\mathrm{CuSO}_{4}$ aqueous solution $(10 \mathrm{~mL}, 40 \mu \mathrm{M})$ was mixed with $\mathrm{BP}$ nanosheets or HABP conjugates solution $\left(10 \mathrm{~mL}, 20 \mu \mathrm{g} \mathrm{mL}^{-1}\right)$ for $1 \mathrm{~h}$. BP nanosheets and HA-BP conjugates were prepared at the desired degradation time point from day 0 to 6. After centrifugation for $15 \mathrm{~min}$ at $12,500 \mathrm{rpm}$, the concentration of metal ions in the supernatants was measured using an atomic absorption spectrometer (TAS-990, Puxi, China).

Binding capacity of BP nanosheets to metal ions Each metal ion stock solution was prepared from $\mathrm{CaCl}_{2}, \mathrm{Mg}\left(\mathrm{NO}_{3}\right)_{2}, \mathrm{ZnCl}_{2}$, $\mathrm{FeCl}_{3} \cdot 6 \mathrm{H}_{2} \mathrm{O}$ and $\mathrm{CuSO}_{4} \cdot 5 \mathrm{H}_{2} \mathrm{O}$, respectively. $\mathrm{BP}$ nanosheets aqueous solution (10 $\mathrm{mL}, 20 \mu \mathrm{g} \mathrm{mL}^{-1}$ ) was mixed with $10 \mathrm{~mL}$ of the metal ion stock solution for $1 \mathrm{~h}$. After centrifugation for $15 \mathrm{~min}$ at $12,500 \mathrm{rpm}$, the concentration of metal ions in the supernatants was measured using an atomic absorption spectrometer (TAS-990, Puxi, China). The binding capacity was calculated by the following equation as previously reported elsewhere [9]:

Binding capacity $=\frac{\mathbf{C}_{\mathrm{T}}-\mathbf{C}_{\mathrm{s}}}{\mathbf{C}_{\mathrm{T}}} \times 100 \%$

where $C_{T}$ represents the total concentration of metal ions in the mixture and $C_{S}$ represents the concentration of metal ions in the supernatant.

Characterization of BP-Cu and HA-BP-Cu complexes

The energy dispersive spectroscopy mapping (EDS mapping) of $\mathrm{BP}, \mathrm{BP}-\mathrm{Cu}, \mathrm{HA}$ $B P, H A-B P-C U$ was performed with a field emission - scanning electron microscope (FE-SEM, JSM-7401F, JEOL, Akishima, Japan).

In vitro biocompatibility test

Liver cancer cells, HepG2, in DMEM were maintained in a humidified $5 \% \mathrm{CO}_{2}$ incubator at $37^{\circ} \mathrm{C}$. HepG2 cells were seeded at a density of $2 \times 10^{4}$ onto 96well plates and treated with different concentrations of BP nanosheets and HABP conjugates for $24 \mathrm{~h}$. Serum-free medium and CCK-8 were added to the cells after washing with PBS. The relative cell viability $(n=3)$ was assessed by the microplate reader (EMax microplate reader, Bucher Biotec AG, Basel, Switzerland).

In vitro cellular uptake

HepG2 cells were seeded at a density of $1 \times 10^{4}$ cells onto the confocal dish. After incubating for a day, the cells were pre-incubated with excess $\mathrm{HA}$ for $2 \mathrm{~h}$ 
to confirm HA receptor-mediated endocytosis of HA-BP-FITC conjugates. BPFITC or HA-BP-FITC conjugates were added, incubated for $2 \mathrm{~h}$, and washed with PBS. The cells were fixed with $4 \%$ paraformaldehyde solution and stained with DAPI. Images were acquired by confocal microscopy (TCS SP5 Ltd., Leica Korea).

\section{Statistical analysis}

Statistical comparison was performed using the software SigmaPlot 10.0 (Systat Software Inc. San Jose, CA). Values for ${ }^{*} P<0.05$ and ${ }^{* *} P<0.001$ were considered significant.

\section{Results}

Preparation and characterization of HA-BP conjugates

BP nanosheets were prepared from bulk BP by liquid exfoliation. Then, HA-BP conjugates were synthesized by simple mixing of BP nanosheets with HA-DAH. $\mathrm{BP}$ nanosheets and the amino groups of HA-DAH were physically bound by electrostatic interaction. DLS revealed the size increase from $226.6 \pm 46.6 \mathrm{~nm}$ to $258.8 \pm 42.3 \mathrm{~nm}$ after mixing of BP nanosheets with HA-DAH (Figure 1a). The zeta potential of HA-BP conjugates $(-37.1 \pm 0.4 \mathrm{mV})$ was increased from -29.6 $\pm 1.1 \mathrm{~nm}$ due to the amine groups of HA-DAH (Figure 1b). There was no obvious change in the absorbance spectra of BP nanosheets after HA coating (Figure 1c). To confirm the successful synthesis of HA-BP conjugates, FT-IR spectroscopy was performed (Figure 1d). FT-IR spectrum of HA-BP conjugates showed noticeably increased peaks of $\mathrm{O}-\mathrm{H} \& \mathrm{~N}-\mathrm{H}$ stretching $\left(3000-3500 \mathrm{~cm}^{-1}\right)$ and amide $\mathrm{C}=\mathrm{O} \& \mathrm{C}-\mathrm{N}$ stretching $\left(1600-1700 \mathrm{~cm}^{-1}\right)$ in HA-BP conjugates. TEM showed that both BP nanosheets and HA-BP conjugates were free-standing with a uniform planar morphology (Figures 1e and f).

Enhanced stability and biodegradation of HA-BP conjugates

$\mathrm{BP}$ nanosheets are known to be decomposed into phosphoric acid (PA) in the presence of oxygen [19]. HA can prevent the rapid degradation of BP nanosheets under oxygen circumstance and aggregation with the adsorption of serum proteins $[20,21]$. To assess the effect of HA on the stability of BP nanosheets, $\mathrm{BP}$ nanosheets and $\mathrm{HA}-\mathrm{BP}$ conjugates at the equal concentration were dispersed in $\mathrm{DI}$ water. As shown in Figure $2 \mathrm{a}$, the absorbance of $\mathrm{BP}$ nanosheets was significantly decreased for 7 days and barely remained at day 6. In contrast, HA-BP conjugates were stable without precipitation and the absorbance spectra was nearly unchanged after 4 days (Figure $2 b$ ). In addition, the enhanced stability of HA-BP conjugates was assessed by the fluorescent intensity of FITC (Figure 2c). In vitro release of FITC indicated that FITC or HAFITC was separated from BP-FITC or HA-BP-FITC conjugates by degradation. The amount of FITC released from HA-BP-FITC conjugates was relatively small with improved stability, and the concentration of FITC was consistent with the degradation rate (Figure $2 a$ and $b$ ). The colour of BP nanosheets solution became thin after 7 days, compared to that of HA-BP conjugates (Figure 2d). 
All these results confirmed that HA efficiently protected BP nanosheets from subsequent degradation.

Copper specific capturing of HA-BP conjugates

The $\mathrm{Cu}^{2+}$ capturing capacity of BP nanosheets and HA-BP conjugates was investigated by mixing their solutions with $\mathrm{Cu}^{2+}$ solution for $1 \mathrm{~h}$, centrifugation, and dispersion in DI water. The TEM images showed that BP nanosheets and HA-BP conjugates maintained their structures after binding with $\mathrm{Cu}^{2+}$ (Figure 3a). After 7 days, the degraded BP nanosheets and the degraded HA-BP conjugates interacted with $\mathrm{Cu}^{2+}$. The TEM images showed that only HA-BP conjugates still maintained the nanosheets structure binding with $\mathrm{Cu}^{2+}$ (Figure 3a). The morphology of BP nanosheets disappeared and the spherical shape was obtained only due to copper [22]. To evaluate the effect of degradation on copper binding capacity, BP nanosheets and HA-BP conjugates were prepared at the desired degradation time point from day 0 to 6 . After mixing the solution of $\mathrm{BP}$ nanosheets or $\mathrm{HA}-\mathrm{BP}$ conjugates with $\mathrm{Cu}^{2+}$ solution for $1 \mathrm{~h}$, the mixture was centrifuged and the concentration of $\mathrm{Cu}^{2+}$ in supernatants was analyzed with an atomic absorption spectrometer. As shown in Figure $3 \mathrm{~b}$, the $\mathrm{Cu}^{2+}$ capturing capacity of BP nanosheets was dramatically decreased for 7 days. In contrast, the copper binding capacity of HA-BP conjugates was only slightly decreased owing to the HA coating, which was well matched with the degradation rate of HA-BP conjugates in Figure $2 \mathrm{~b}$.

Meanwhile, the copper specific binding of BP nanosheets and HA-BP conjugates was assessed with an atomic absorption spectrometer by comparing the intensity change of each metal ion before and after mixing for 1 h (Figure $3 \mathrm{c}$ ). BP nanosheets and HA-BP conjugates selectively interacted with $\mathrm{Cu}^{2+}$, which indicated the excellent copper capturing specificity. The concentration of other metal ions, such as $\mathrm{Ca}^{2+}, \mathrm{Mg}^{2+}$, and $\mathrm{Zn}^{2+}$ was nearly unchanged. The reason might be that the Gibbs free energy change $(\triangle G)$ of BP$\mathrm{Cu}$ or HA-BP-Cu complex was much lower than that of BP-metal or HA-BPmetal complexes [9]. The binding capacity of HA-BP conjugates to $\mathrm{Fe}^{2+}$ was slightly increased because of chelating effect of HA [23]. In addition, the copper capturing capacity of HA-BP conjugates was increased through the chelatormetal complex via amine groups of HA-DAH [6].

We next investigated the distribution of $\mathrm{Cu}^{2+}$ on BP nanosheets and HA-BP conjugates by the FE-SEM element mapping (Figure 4). The $C$ element EDS mapping was only observed in the HA-BP conjugates because of HA carbon chains. Copper ions were co-localized with $P$ elements on the EDS mapping of $\mathrm{BP}-\mathrm{Cu}$ and HA-BP-Cu complexes. These results successfully demonstrated the high capturing capacity of HA-BP conjugates to $\mathrm{Cu}^{2+}$. 
The cellular uptake of BP-FITC and HA-BP-FITC conjugates into the liver cancer cells, HepG2, was investigated by confocal microscopy. HA-BP-FITC conjugates only showed green fluorescence of FITC, indicating the liver cell targeted delivery of HA-BP conjugates (Figure $5 \mathrm{a}$ ). HA interacts with liver cells via HAbiding receptors, such as receptor for HA-mediated motility (RHAMM) and CD44 [20, 24]. To study the cellular uptake mechanism of HA-BP-FITC conjugates, HepG2 cells were pre-treated with excess HA. The fluorescence of $\mathrm{HA}-\mathrm{BP}$-FITC conjugates was reduced in the cells pre-incubated with HA, which revealed that the uptake of HA-BP-FITC conjugates was mediated by HA receptor-mediated endocytosis. The in vitro biocompatibility of $\mathrm{BP}$ nanosheets and HA-BP conjugates was analyzed by CCK-8 assay. As shown in Figure $5 b$, the viability of HepG2 cells after treatment with HA-BP conjugates was slightly higher than that with BP nanosheets, which might be ascribed to the biocompatible HA coating $[25,26]$.

\section{Discussion}

This study aimed to protect BP nanosheets from rapid degradation in oxygen circumstances and increase copper binding capacity for WD treatment. Previous studies have reported limitations of BP nanosheets in biomedical applications due to the rapid degradation of BP nanosheets [13, 19]. As shown in Figure 2 and 3, BP nanosheets were rapidly decomposed into phosphorus atoms and copper binding capacity also decreased with the degree of decomposition. To overcome this issue, BP nanosheets were coated with various polymers such as polyethylene glycol (PEG), HA, and poly lactic-coglycolic acid (PLGA) [27, 28]. However, there are no previous studies of the change in the copper binding capacity of BP nanosheets after surface modification. Also, although the liver is a major organ where excess copper accumulates in WD, there are few in-depth studies on delivering chelating agents to the liver for the treatment of WD. In this research, BP nanosheets were modified with $\mathrm{HA}$ for improved stability and targeted delivery to the liver. HA has been widely investigated in the biomedical field due to its excellent biocompatibility and targeted delivery to the liver or tumor [17, 18, 20]. Our results demonstrated that $\mathrm{HA}$ successfully prevented $\mathrm{BP}$ nanosheets from rapid degradation and maintained the $\mathrm{Cu}^{2+}$ capturing capacity of BP nanosheets after degradation for 7 days (Figure 2 and 3). The most significant implication was that the copper binding capacity of HA-BP conjugates was higher than that of BP nanosheets, as shown in Figure $3 \mathrm{~b}$ and $\mathrm{c}$. Amine groups of HA-DAH can react with transition metals via chelator-metal complex, such as $\mathrm{Fe}^{2+}$ and $\mathrm{Cu}^{2+}$, and may increase copper binding capacity [6]. Moreover, the cellular uptake of HA-BP conjugates into the liver cancer cells, HepG2, demonstrated the liver cell targeted delivery of HA-BP conjugates (Figure 5a). Although the exact mechanism underlying the improved copper capturing capacity of HA-BP conjugates was not evaluated in this study, our results sufficiently showed 
enhanced stability, copper-specific binding capacity, and hepatic targeted delivery of HA-BP conjugates. This study could be further processed for in vivo animal studies of WD treatment, as schematically shown in Figure 6.

\section{Conclusion}

We have successfully developed HA-BP conjugates as a copper specific chelating agent for the treatment of WD. HA-BP conjugates could selectively capture $\mathrm{Cu}^{2+}$ among various transition-metal ions, such as $\mathrm{Ca}^{2+}, \mathrm{Mg}^{2+}, \mathrm{Fe}^{2+}$, and $\mathrm{Zn}^{2+}$. The copper binding capacity of HA-BP conjugates was significantly higher than that of BP nanosheets. In addition, $\mathrm{HA}$ was able to prevent BP nanosheets from rapid degradation under oxygen environment and maintain the $\mathrm{Cu}^{2+}$ capturing capacity of HA-BP conjugates. With enhanced stability, HA-BP conjugates showed excellent biocompatibility in HepG2 cells. In vitro cellular uptake test with HepG2 cells confirmed the feasibility for targeted delivery of HA-BP conjugates to the liver. Taken together, HA-BP conjugates would be a promising liver targeting and copper chelating agent for WD treatment. 
Acknowledgements

The research was supported by the Basic Science Research Program (2020R1A2C3014070) and the Korea Medical Device Development Fund grant (2020M3E5D8105732).

Funding

This work was supported by the grant code: 2020R1A2C3014070, 2020M3E5D8105732.

Availability of data and materials

All data analyzed in this study are included in this published article.

Ethics approval and consent to participate

N/A

Competing interests

The authors declare that they have no competing interests.

Consent for publication

The manuscript has been submitted with the consent of all authors for publication.

Authors' contributions

S.-J.K. and H.H.H contributed towards the conception and design of this study, acquisition of data, analysis and drafting of the article. H.S.K. conceived of the study, designed the study, coordinated the study and helped draft the manuscript. All authors gave final approval for publication and agree to be held accountable for the work performed therein.

References

1. Ala, A., Walker, A.P., Ashkan, K., Dooley, J.S., Schilsky, M.L.: Wilson's disease. Lancet. 369, 397-408 (2007)

2. Petrasek, J., Jirsa, M., Sperl, J., Kozak, L., Taimr, P., Spicak, J., Filip, K., Trunecka, P.: Revised King's College score for liver transplantation in adult patients with Wilson's disease. Liver Transpl. 13, 55-61 (2007)

3. Roberts, E. A., Schilsky, M.L.: Diagnosis and treatment of Wilson disease: an update. Hepatology 47, 2089-2111 (2008)

4. Askari, F.K., Greenson, J., Dick, R.D., Johnson, V.D., Brewer, G.J.: Treatment of Wilson's disease with zinc. XVIII. Initial treatment of the hepatic decompensation presentation with trientine and zinc. J. Lab. Clin. Med. 142, 385390 (2003)

5. Santos, S.E.E.; Sarles, J.; Buts, J.P., Sokal, E.M.: Successful medical treatment of severely decompensated Wilson disease. J. Pediatr. 128, 285-287 (1996)

6. Członkowska, A., Litwin, T.: Wilson disease - currently used anticopper therapy. Hnadb. Clin. Neurol. 142, 181191 (2017)

7. Medici, V., Trevisan, C.P., D'Incà, R., Barollo, M., Zancan, L., Fagiuoli, S., Martines, D., Irato, P., Sturniolo, G.C.: Diagnosis and management of Wilson's disease: results of a single center experience. J. Clin. Gastroenterol 40, 936-941(2006)

8. Walshe, J.M.: Wilson's disease presenting with features of hepatic dysfunction: a clinical analysis of eighty-seven patients. Q. J. Med. 70, 253-63 (1989)

9. Chen, W., Quyang, J., Yi, X., Xu, Y., Niu, C., Zhang, W., Wang, L., Sheng, J., Deng, L., Liu, Y.N., Guo, S.: Black Phosphorus Nanosheets as a Neuroprotective Nanomedicine for Neurodegenerative Disorder. Adv. Mater. 30, $1703458(2018)$

10. Liu, G., Men, P., Perry, G, Smith, M.A.: Metal chelators coupled with nanoparticles as potential therapeutic agents for Alzheimer's disease. J. Nanoneurosci. 1, 42-55 (2009)

11. Kwon, H., Seo, S.W., Kim, T.G., Lee, E.S., Lanh, P.T., Yang, S., Ryu, S., Kim, J. W.: Ultrathin and Flat Layer Black Phosphorus Fabricated by Reactive Oxygen and Water Rinse. ACS Nano 10, 8723 (2016)

12. Zhang, T., Wan, Y., Xie, H., Mu, Y., Du, P., Wang, D., Wu, X., Ji, H., Wan, L.: Degradation Chemistry and Stabilization of Exfoliated Few-Layer Black Phosphorus in Water. J. Am. Chem. Soc. 140, 7561-7567 (2018)

13. Choi, J.R., Young, K.W., Choi, J.Y., Nilghaz, A., Lin, Y., Xu, J., Lu, X.: Black Phosphorus and its Biomedical Applications. Theranostics 8, 1005-1026 (2018)

14. Li, Y., Feng, P., Wang, C., Miao, W., Huang, H.: Black phosphorus nanophototherapeutics with enhanced stability and safety for breat cancer treatment. Chemical Engineering Journal 400, 125851 (2000)

15. Kim, H., Jeong, H., Han, S., Beack, S., Hwang, B.W., Shin, M., Oh, S.S., Hahn, S.K.: Hyaluronate and its derivatives for customized biomedical applications. Biomaterials 123, 155-171 (2017)

16. Kim, H., Shin, M., Han, S., Kwon, W., Hahn, S.K.: Hyaluronic Acid Derivatives for Translational Medicines. Biomacromolecules 20, 2889-2903 (2019)

17. Lee, M.Y., Yang, J.A., Jung, H.S., Beack, S., Choi, J.E., Hur, W., Koo, H., Kim, K., Yoon, S.K., Hahn, S.K: Hyaluronic acid-gold nanoparticle/interferon a complex for targeted treatment of hepatitis $\mathrm{C}$ virus infection. ACS Nano 6, 9522-9531 (2012)

18. Oh, E.J., Park, K.T., Kim, K.S., Kim, J.S., Yang, J.A., Kong, J.H., Lee, M.Y., Hoffman, A.S., Hahn, S.K.: Target Specific and Long-Acting Delivery of Protein, Peptide, and Nucleotide Therapeutics Using Hyaluronic Acid Derivatives. J. Control. Rel. 141, 2-12 (2010)

19. Zhou, Q., Chen, Q., Tong, Y., Wang, J.: Light-induced ambient degradation of few layer black phosphorus: mechanism and protection. Angew. Chem. Int. Ed. 55, 11437-11441 (2016)

20. Mattheolabakis, G., Milane, L., Singh, A., Amiji, M.M.: Hyaluronic acid targeting of CD44 for cancer therapy: from receptor biology to nanomedicine. J. Drug Target. 23, 605-618 (2015)

21. Pinto, A.M., Moreira, J.A., Magalhaes, F.D., Goncalves, I.C.: Polymer surface adsorption as a strategy to improve the biocompatibility of graphene nanoplatelets. Colloids Surf. B 146, 818-824 (2016)

22. Cuevas, R., Durán, N., Diez, M.C., Tortella, G.R., Rubilar, O.: Extracellular Biosynthesis of Copper and Copper Oxide Nanoparticles by Stereum hirsutum, a Native White-Rot Fungus from Chilean Forests. J. Nanomater. 10, 1-7 (2015)

23. Balogh, G.T., Illés, J., Sézkely, Z., Forrai, E., Gere, A.: Effect of different metal ions on the oxidative damage and antioxidant capacity of hyaluronic acid. Arch. Biochem. Biophys. 410, 76-82 (2003)

24. He, X., Nie, N., Wang, K., Tan, W., Wu, X., Zhang, P.: In vivo study of biodistribution and urinary excretion of surface-modified silica nanoparticles. Anal. Chem. 80, 9597-9603 (2008)

25. Kim, K.S., Kim, Y.S., Bao, K., Wada, H., Choi, H.S., Hahn, S.K.: Bioimaging of Botulinum Toxin and Hyaluronate Hydrogels Using Zwitterionic Near-Infrared Fluorophores. Biomater Res. 21, 15 (2017)

26. Yeom, J.S., Hwang, B.W., Yang, D.J., Shin, H.I., Hahn, S.K.: Effect of Osteoconductive Hyaluronate Hydrogels on Calvarial Bone Regeneration. Biomater Res. 18, 8 (2014)

27. Tao, W., Zhu, X., Yu, X., Zeng, X., Xiao, Q., Zhang, X., Ji, X., Wang, X., Shi, J., Zhang, H., Mei, L.: Black Phosphorus Nanosheets as a Robust Delivery Platform for Cancer Theranostics. Adv. Mater. 29, 1603276 (2017)

28. Zeng, G., Chen, Y.: Surface modification of black phosphorus-based nanomaterials in biomedical applications: Strategies and recent advances. Acta Biomaterialia 118, 1-17 (2020) 
(a)

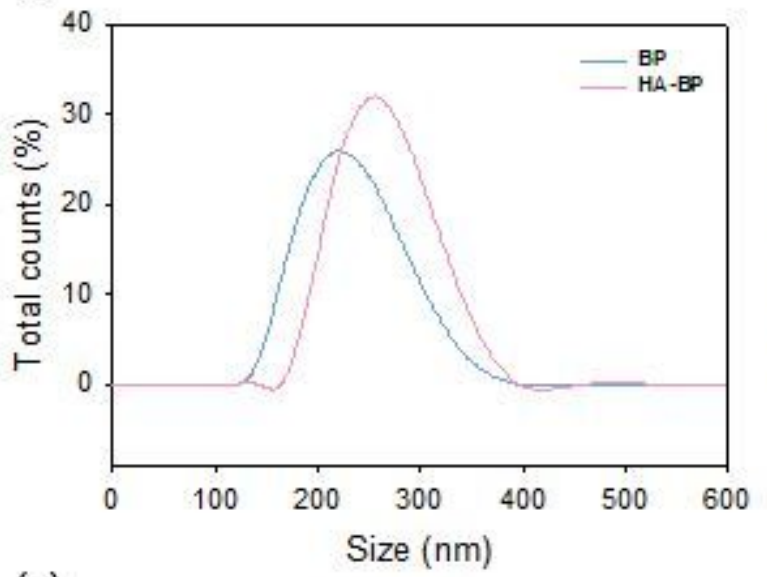

(c)

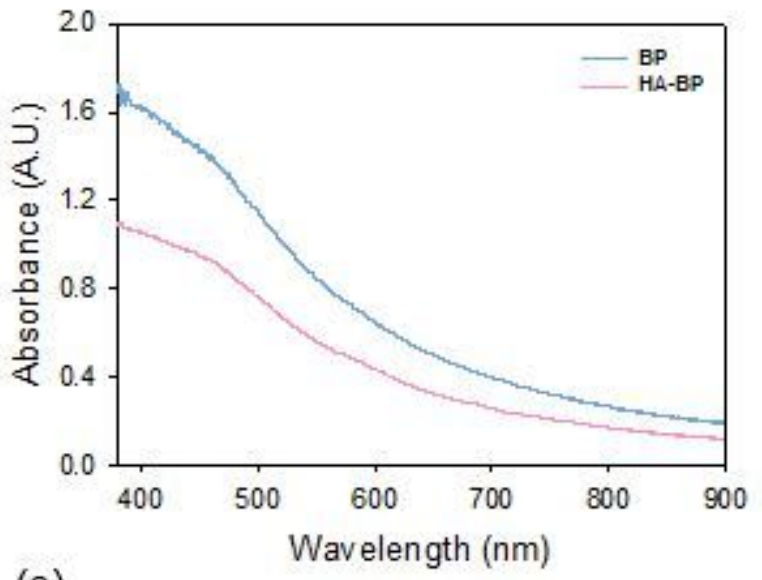

(e)

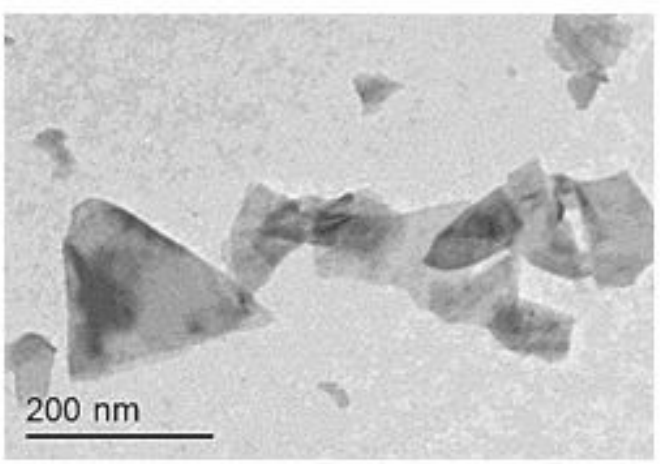

(b)

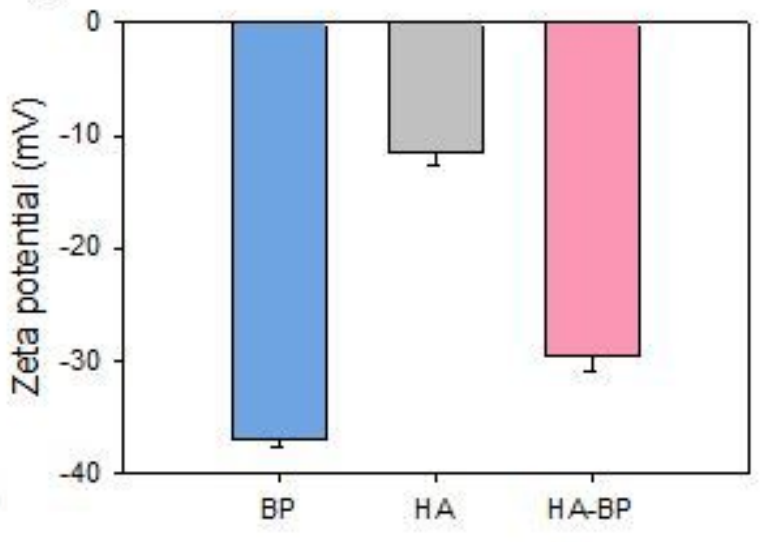

(d)

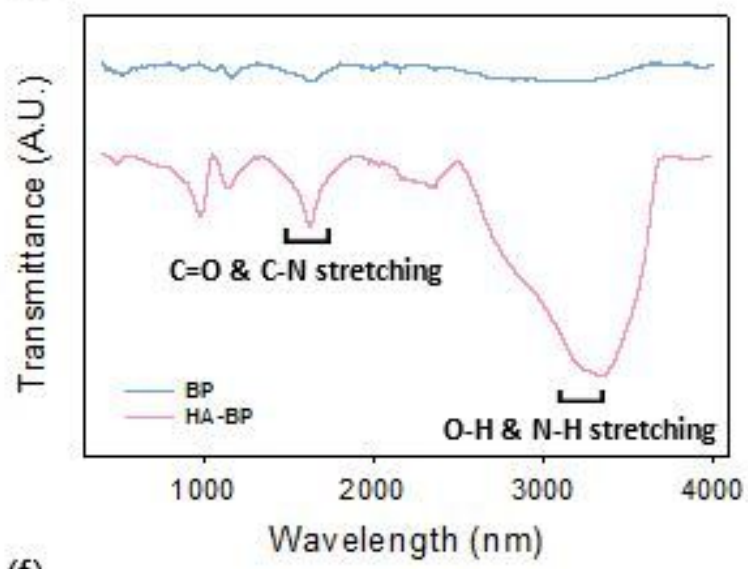

(f)

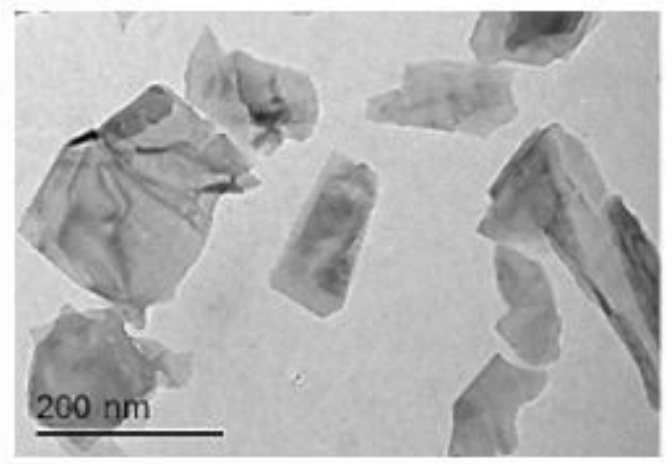

Figure 1

Characteristics of BP nanosheets and HA-BP conjugates. (a) Size distribution, (b) zeta potential, (c) absorbance spectra and (d) FT-IR spectra of BP nanosheets and HA-BP conjugates. TEM images of (e) $\mathrm{BP}$ nanosheets and (f) HA-BP conjugates. Data are expressed as mean \pm SD. 
(a)

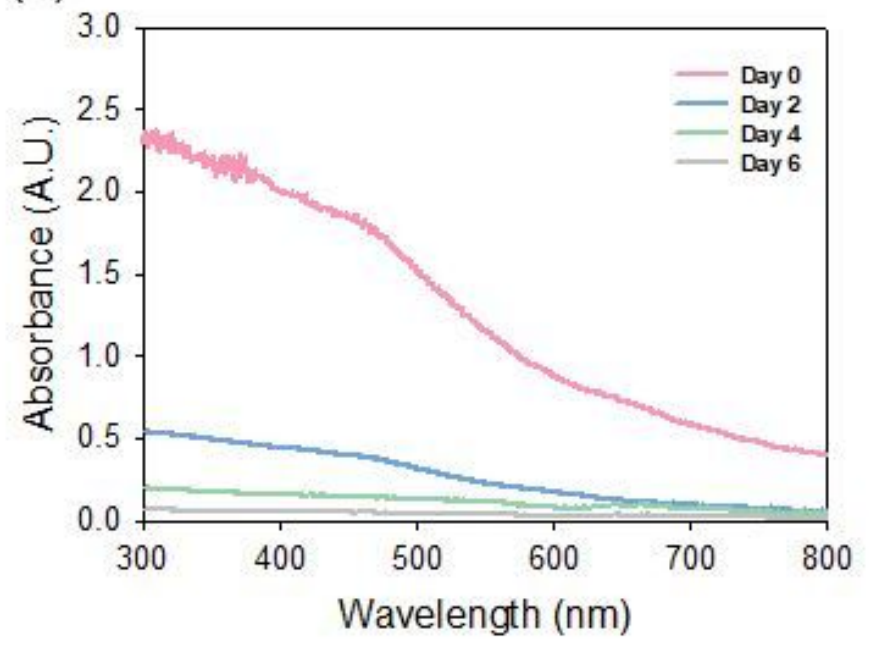

(c)

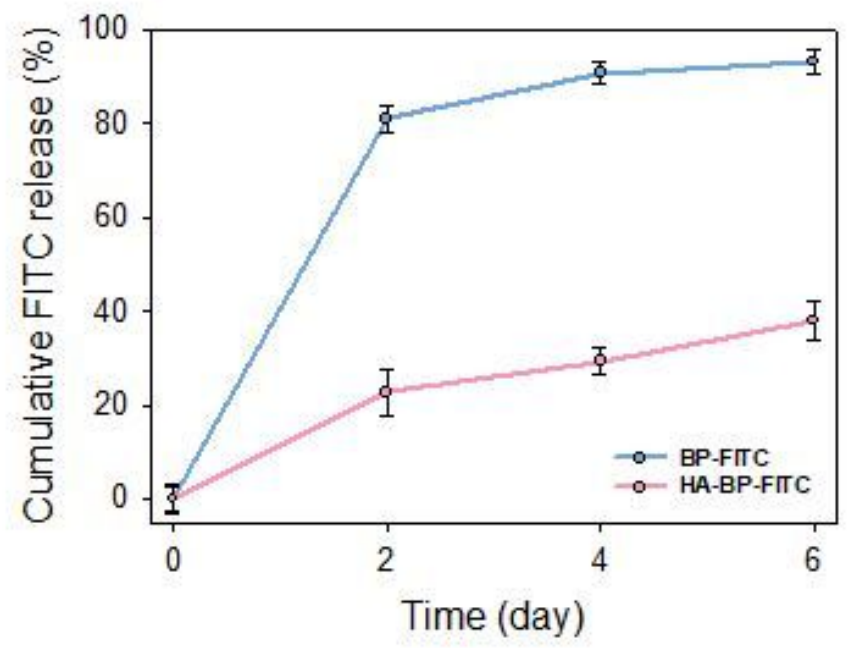

(b)

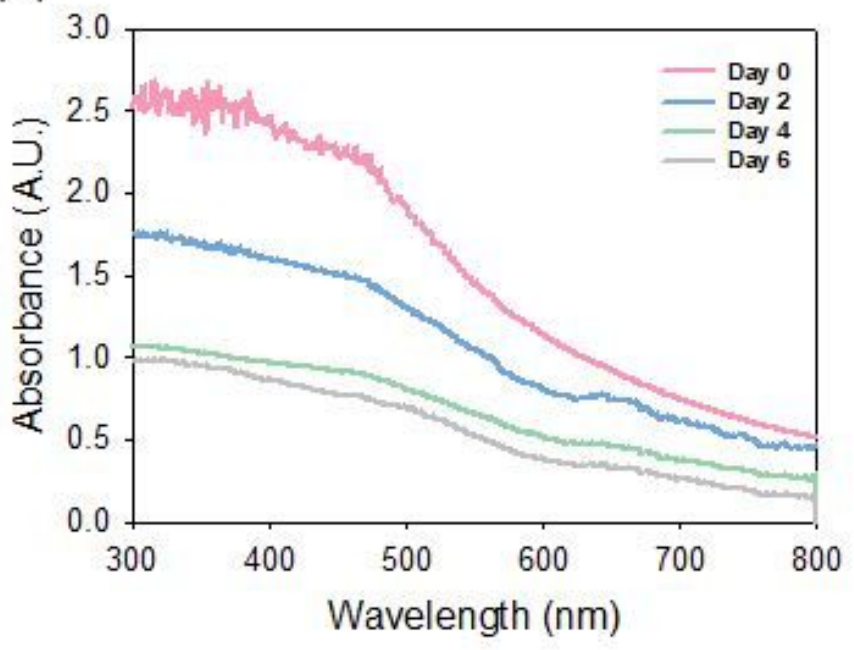

(d)

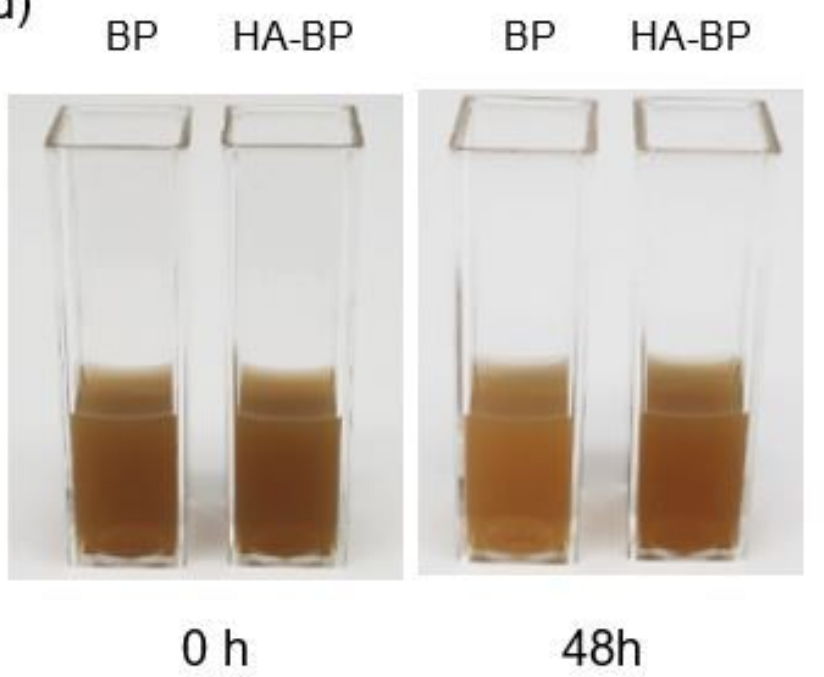

\section{Figure 2}

Stability of HA-BP conjugates. Absorbance spectra of (a) BP nanosheets and (b) HA-BP conjugates in DI water for 7 days. (c) In vitro release of FITC from BP-FITC and HA-BP-FITC conjugates in DI water for a week $(n=3)$. (d) Photographs of BP nanosheets and HA-BP conjugates in DI water for $48 \mathrm{~h}$. 
(a)

\section{BP-Cu}
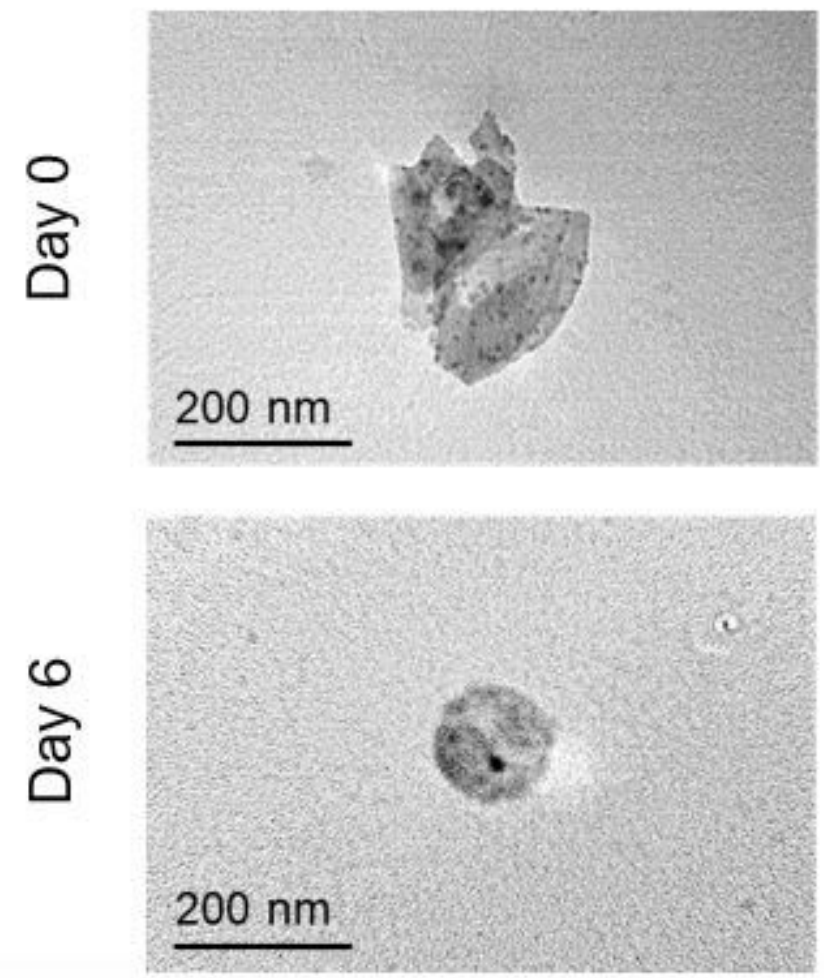

(b)

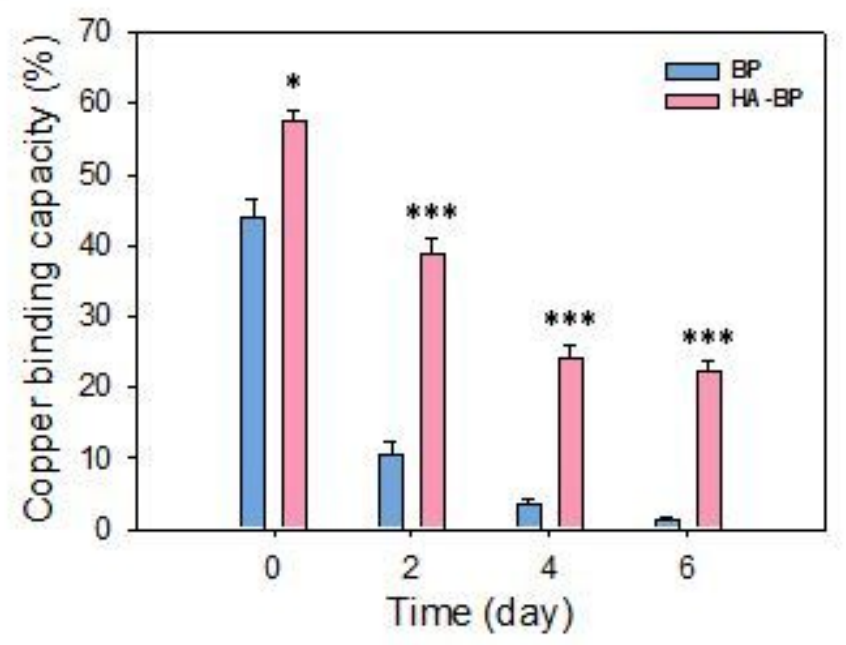

HA-BP-Cu
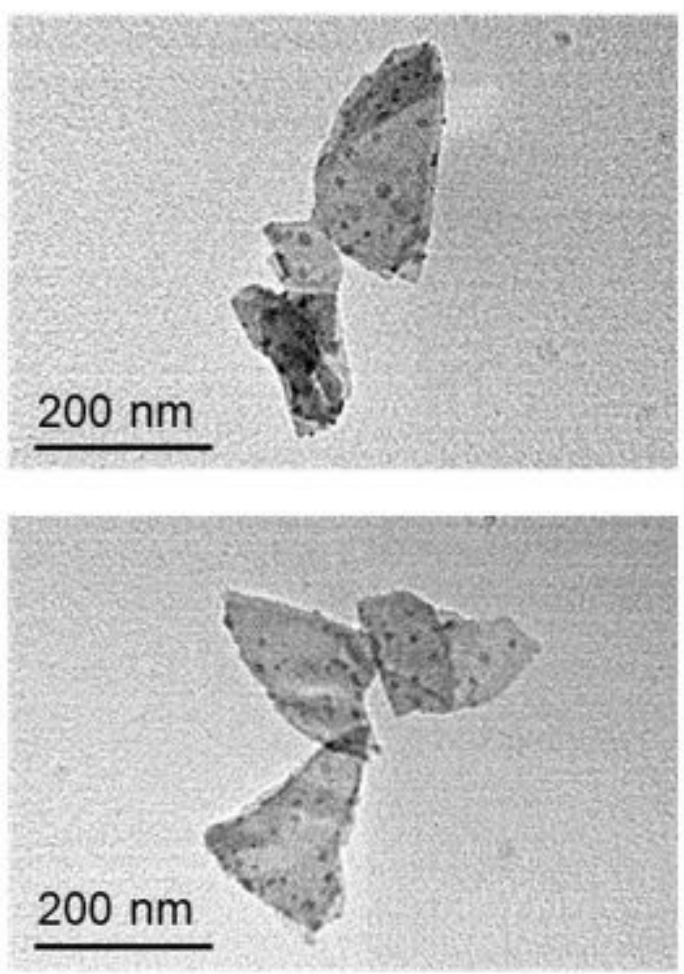

(c)

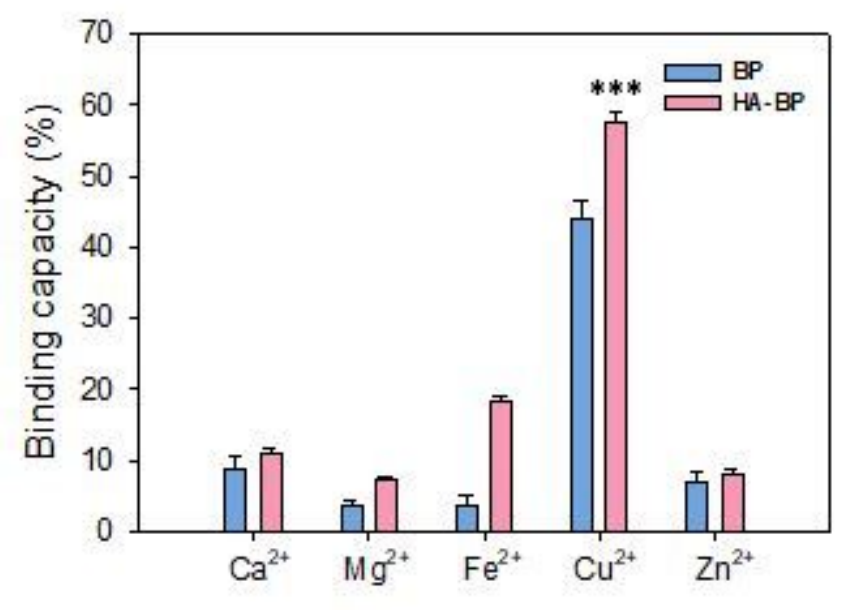

Figure 3

Copper capturing capacity of HA-BP conjugates. (a) TEM images of BP-Cu and HA-BP-Cu complexes after degradation of BP nanosheets and HA-BP conjugates for 7 days. The binding capacity of BP nanosheets and HA-BP conjugates to (b) copper ions after degradation of BP nanosheets and HA-BP conjugates for 7 days ( $n=3,{ }^{*} P<0.05, \star \star \star P<0.001$, HA-BP conjugates versus BP nanosheets) and (c) different metal ions after mixing for $1 \mathrm{~h}(\mathrm{n}=3, \star \star * P<0.001$, copper ion versus other metal ions for HA-BP conjugates). 


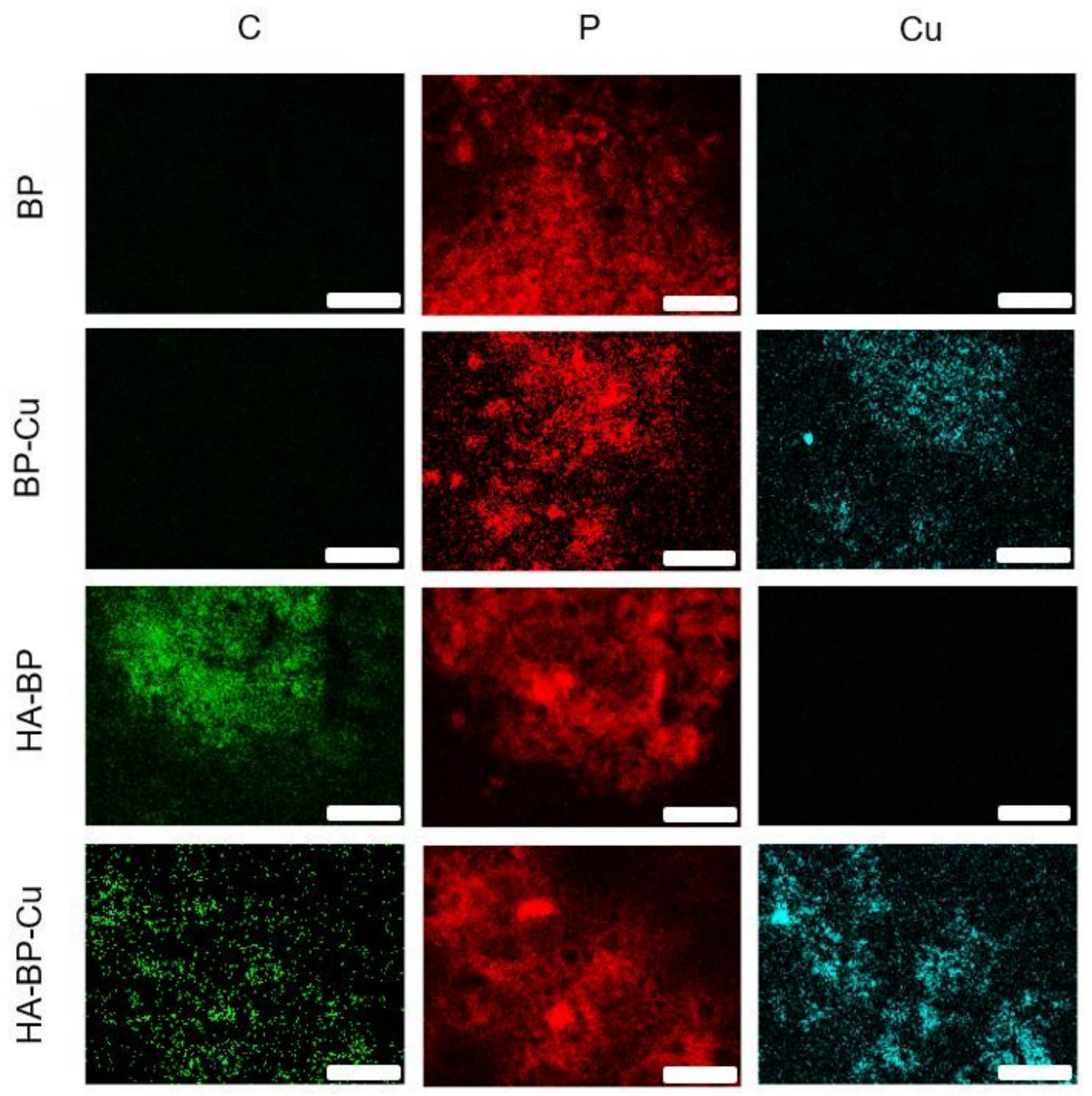

Figure 4

FE-SEM elemental mapping of C, P, and Cu for BP nanosheets, BP-Cu, HA-BP, and HA-BP-Cu complexes (scale bar $=2.5 \mu \mathrm{m})$. 
(a)

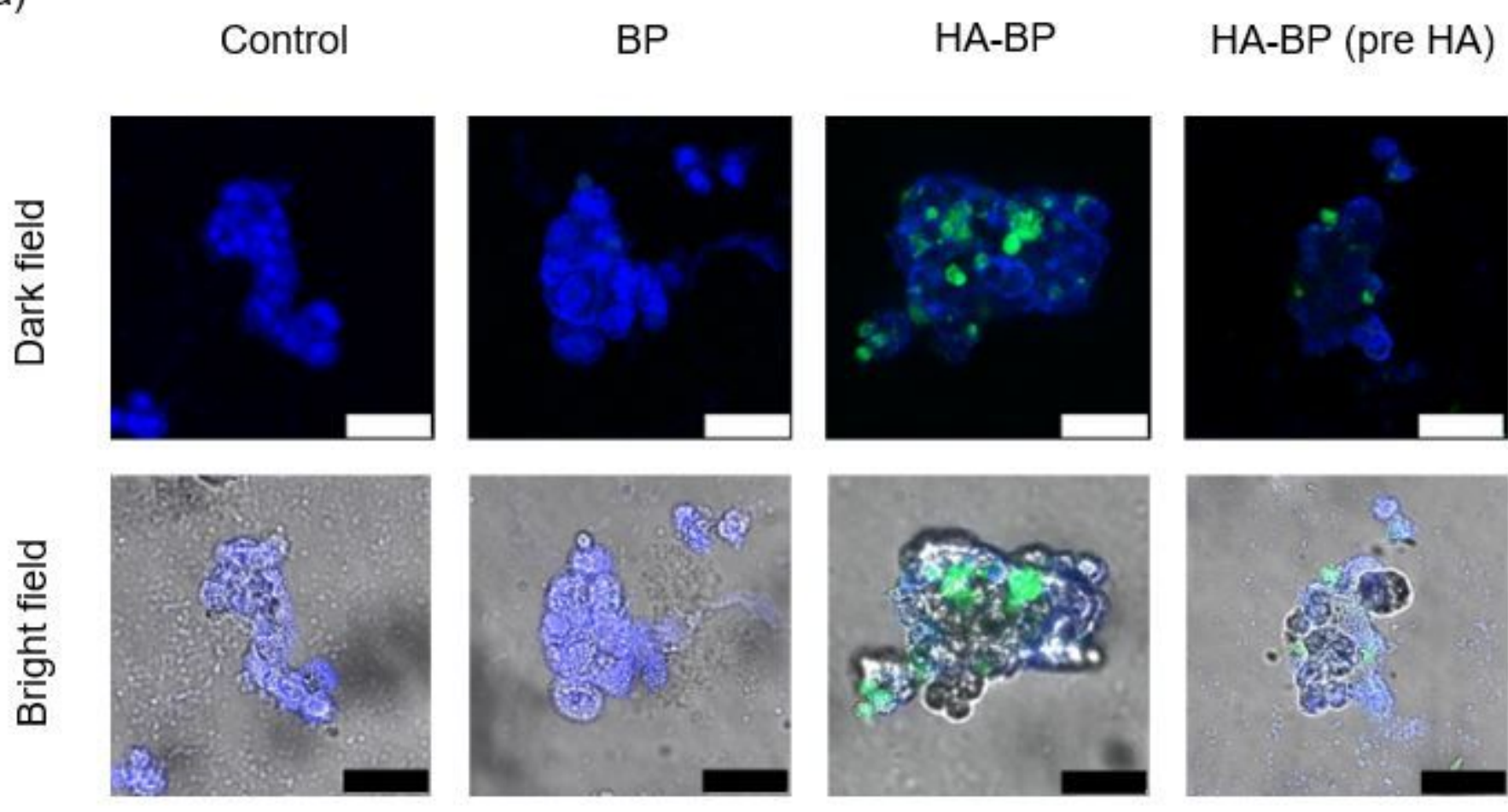

(b)

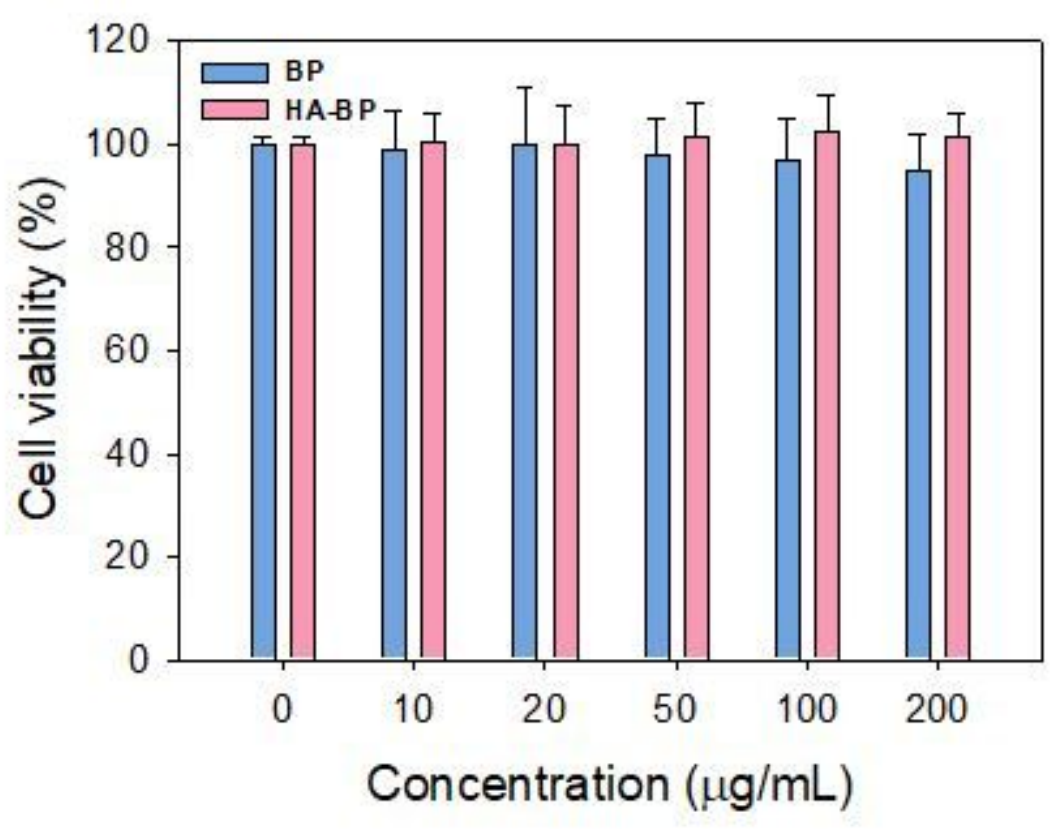

Figure 5

Cellular uptake and biocompatibility assessment of HA-BP conjugates. (a) Confocal microscopy of HepG2 cells after treatment with BP-FITC and HA-BP-FITC conjugates (scale bar $=50 \mu \mathrm{m}$ ) without and with HA pre-incubation (Blue: DAPI and Green: FITC). (b) The biocompatibility of BP nanosheets and HABP conjugates in HepG2 cells by the CCK-8 assay $(n=4)$. Data are expressed as mean \pm SD. 


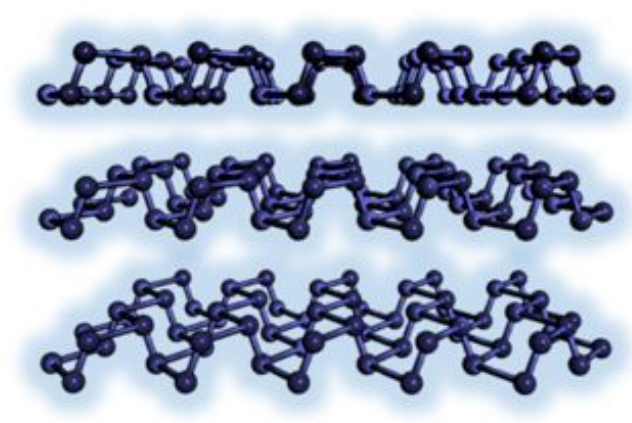

BP nanosheet

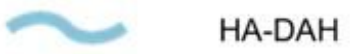

HA-BP conjugate

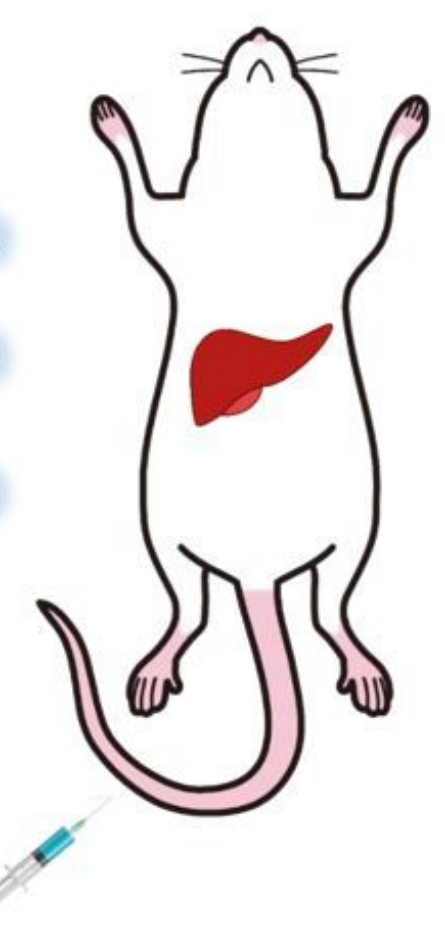

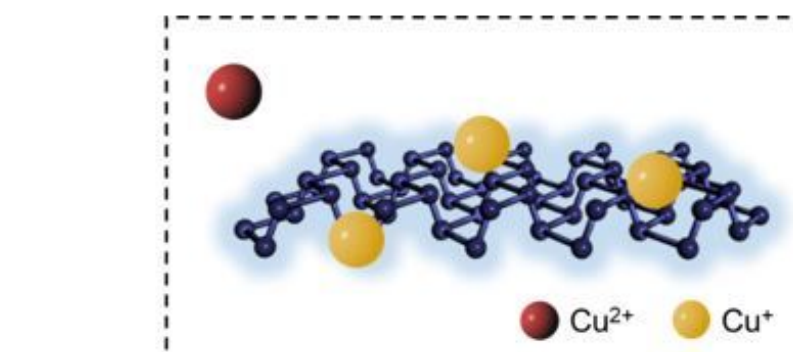

HA-BP-Cu complex

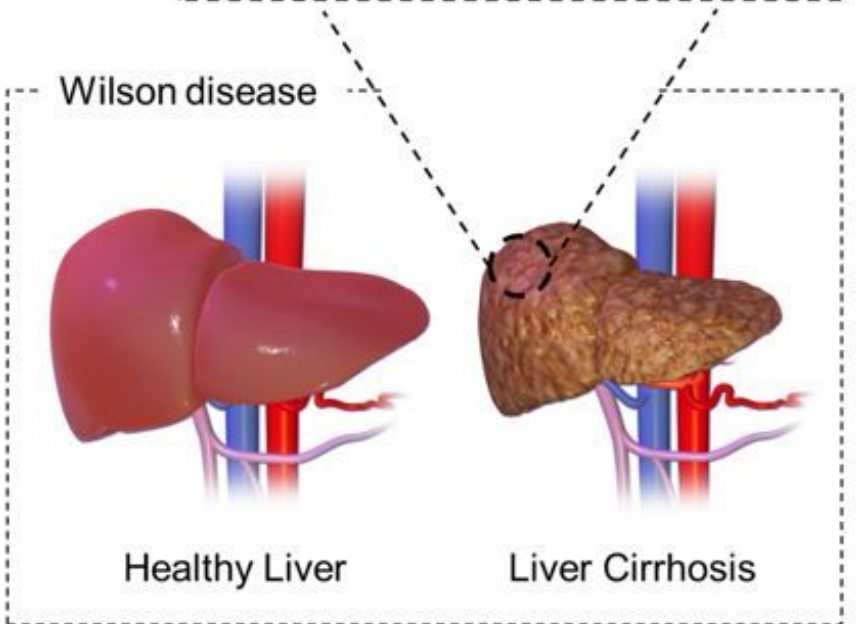

Figure 6

Schematic illustration of HA-BP conjugates as a copper chelating agent to remove copper ions accumulated in the liver for WD treatment. 